American Journal of Applied Sciences 9 (9): 1435-1442, 2012

ISSN 1546-9239

(C) 2012 Science Publication

\title{
Development of Priority Oriented Scheduling Method to Increase the Reliability of Manufacturing Systems
}

\author{
Seyedehfarzaneh Nojabaei, Z. Leman, S.H. Tang and S. Sulaiman \\ Department of Mechanical and Manufacturing Engineering, \\ Faculty of Engineering, UPM Serdang, Malaysia
}

\begin{abstract}
Scheduling occurs in every organization without considering the nature of its activities. In this regard, numerous scholars have attempted to schedule via divergent methods including classical scheduling, genetic algorithm, neural network and fuzzy logic and so on. Studies in manufacturing scheduling mostly deal with priority rules without any consideration of the system states. An appropriate scheduling leads to significant enhancement of fairness in job scheduling. The term fairness can be transformed into a specific selection of job weights. There is no method of scheduling in which priority, time action (duration) and time stamp of jobs have simultaneously been considered. But the proposed method of scheduling can enhance the efficiency and reliability of manufacturing systems. To fulfill this target, first and foremost, the normalize method should be performed. This method allows data (time stamp, time action, priority) of jobs on different scales to be compared by bringing them to a common scale. Secondly, the jobs should be arranged based on three criteria which are priority, time action and time stamp. This sorting algorithm is programmed via MATLAB Distributed Computing Server (DCS) software. Eventually, to evaluate the proposed method of scheduling, simulation is operated. The simulated algorithm shows that applying the proposed method of scheduling increases the efficiency of simulated scheduler in comparison with applying the common method of scheduling. Besides the mentioned simulated algorithm, there is a mathematical proof to show the enhancement of reliability.
\end{abstract}

Key words: Scheduling, distributed control system, manufacturing systems, reliability, efficiency

\section{INTRODUCTION}

Scheduling includes clarifying plan and priority of the jobs which should be performed in a predetermined operation. It means that within an organization, scheduling pertains to establish the time of performing particular resources in the system. Scheduling is directly related to the use of equipment, facilities and human activities. Thus, appropriate scheduling makes efficient use of the capacity (Taiwo, 2007).

Baker (1974) proposed that scheduling occurs in every organization without considering the nature of the organization activities. Manufacturers should plan production by developing schedule for labors, equipment, procurement, design, manufacturing, industrialization, maintenance, even after-sale services and. The scheduling must be designed in a way to keep processors busy by efficiently distributing the workload, usually in terms of response time, resource availability and maximum throughput of application (Samreen and Khiyal, 2007).
In this regard, numerous of scholars have made attempts to schedule via divergent methods includingclassical scheduling (Zhang, 2010), genetic algorithm (Taghavifard et al., 2009; Oguz and Ercan, 2005), neural network artificial (Venkatachalam et al., 2008), fuzzy logic (Omar, 2007; Vinod and Sridharan, 2008), programming model (Chen, 2010) and so on. Kapanoglu and Alikalfa (2011) proposed that scheduling is an indispensable task for manufacturing systems in today's harsh competitive markets. Ever-soaring massive numbers of research efforts have investigated the solution to scheduling problems. No need to say that scheduling problems cannot be solved optimally for even modest problem sizes. Pinedo (2008) described thatscheduling problems cope with the allocation of resources (material, labor, technology) to carry out a set of activities during a period of time.

Studies in manufacturing scheduling mostly deal with priority rules without any consideration of the system states, sometimes due to ease of use in the shop floor. In traditional manufacturing systems, scheduling

Corresponding Author: Seyedehfarzaneh Nojabaei, Department of Mechanical and Manufacturing Engineering,

Faculty of Engineering, UPM Serdang, Malaysia 
is carried out by machine operators and shop supervisors (Kapanoglu and Alikalfa, 2011). Therefore, serious surveillance problems can occur as a result of complex, multi-attribute, or state-observing rules. Computer-Integrated Manufacturing (CIM) systems, on the other hand, do not face these sorts of challenges (Mahdavi and Shirazi, 2010).

A priority rule is used to determine which job from a queue is to be operated next. Numerous priority rules have been introduced in the literature. The priorityscheduling algorithm with much more reduced waiting time for the processes (Rashid and Akhtar, 2006).

Davis (1987) compared the performance of often individual priority rules with a randomized combination of these rules and presented the combined method provides far superior results, but requires substantially more computing time. No priority rule appears to be superior to all the others (Pierreval and Mebarki, 1997). Also, the best priority rule cannot be known as a priori several times. Some researchers adopt simulation and determine the most favorable priority rule(s) for the operating conditions, production objectives and current shop status (Mebarki et al., 1998).

According to the definition of reliability, the reliability of production scheduling process refers to the probability of completing the prescribed functions on the prescribed conditions and during the regulated working time. Reliability is the probability that a system conducts a particular service during a specified period of time (Wu and Zuo, 2010). Reliability analysis banks on stochastic models of the frequency, interval and intensity of faults in hardware and software (Smith, 2005). Adamyan and He (2002) stated one of the most substantial concepts in manufacturing industries is to assess of reliability and safety with sequential failures. Ishikawa (1985) defined each cause or reason for fault is a source of variation. Causes are commonly classified into chief categories to recognize these sources of variation. The categories typically include people, methods, machines, material, management and the environment in which faults can be classified. Adamyan and $\mathrm{He}$ (2002) held a view that not only the safety and reliability of systems are directly dependent on all failure states of system but also depend on the sequential occurrences of those failures, it means that the priority of jobs to perform are influential on reliability of manufacturing systems. In order to quantify system security and the grave consequence of accidental failures, several researches have been carried out (Huafei et al., 2006; Sheyner et al., 2002).

The other important point to mention is that applying appropriate method of scheduling causes significant enhancement of fairness in job scheduling.
Among all jobs, scheduling can also provide very good overall performance (Sabin et al., 2007). Furthermore, this scheduling can decrease the number of failures and increase fairness in job assigning. Fairness can be defined as a powerful abstraction that has led to utilitarian results in manufacturing systems. Krallmann et al. (1999) demonstrated that the term fairness can be transformed into a specific selection of job weights. Qiao and Qiaoyun (2009) stated that several operations can be simultaneously processed by one machine with different priorities and jobs with higher priority may be processed earlier. Tian et al. (2009) proposed that a control point is not the timing of a job being processed, but the priority of that job. To model the problem, stochastic programming has been applied by Fazlollahtabar and Zandieh (2010). Shukla et al. (2008) suggests that job scheduling problem can be viewed as an optimization problem, bounded by both sequence and resource constraints. In this study, it is assumed that each job should be performed. Unfair judgment in job scheduling and ignoring the priority of jobs based on the criterion of how much the jobs are tense, can drastically lead into a reduction in the reliability of the whole system.

Problem statement: In accord with the literature on scheduling, there is no method in which all the priority resource criteria, time stamp and time action have simultaneously been considered. Therefore, a more realistic scheduling model should be taken into account of the six effective criteria including machine, maintenance, process, environment, management and material activities. In this regard, the present study focuses on decreasing the probability of crash in manufacturing systems. Besides, in terms of importance and tensing, the time stamp (time of arrival), the time action (duration time), the specific priority (the six above-mentioned criteria) of each job are considered.

Holmes (1995) and Cruz and Tasi (1996) are the first to have hinted at the idea of First-In-First-Out (FIFO). This policy is being applied to the vast majority of manufacturing systems. Due to allocating jobs with FIFO policy and only based on considering the mere criterion of time stamp, the optimizations models have never been fair-oriented. This concept can be quite clarified by supposing that there are three jobs in a paint shop of car manufacturing company referring to a scheduler in a central control room. The first job is to close the ramp door; the second is to move one of the horizontal machine's axis; and the third one is to take action on a message about temperature raise in the mixing room of paint shop. The last one can result in a fire in the mixing room if the immediate measures are not taken. FIFO policy demands those three jobs to be processed just based on time stamp, i.e., the time of 
receiving. However, both first and the second job are time consuming. Therefore, the shop floor might catch fire when the third job is being processed and activated. However, it is obvious that by considering priority aspect for sorting the jobs to process, the probability of catching fire will be decreased or even eliminated. Unfortunately, as long as the only criterion of allocating jobs to appropriate machines is FIFO policy, it can result in decreasing the reliability of the systems in which faults occur. Thus, in this study, three criteria (priority, time action and time stamp) of each job are being simultaneously considered for job scheduling problem.

Proposed scheduling method: As mentioned before, in majority of manufacturing systems, the only criterion of allocating jobs to appropriate machines by schedulers of CCR (central control room) is FIFO policy. Accordingly, considering only the criterion of time stamp is the major cause of failing to provide for fairness in distributed systems. In order to increase the reliability of manufacturing system, the three criteria of time stamp, priority and time action for each job are simultaneously considered in this study. The methodology involves normalizing the data and then sorting jobs via developing bubble sorter in MATLAB distributed computing software. The research is performed on the basis of the criteria of priority, time action duration as well as time stamp. In order to evaluate the proposed method and increase of efficiency, a simulation is created. Then, mathematical proof is provided to show the enhancement ofreliability.

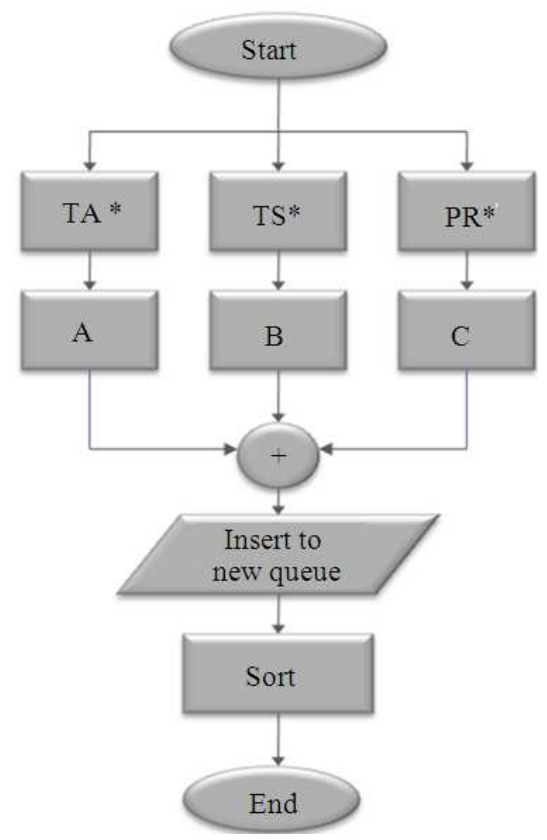

Fig. 1: Flowchart sorting algorithm
Normalize method: Normalization refers to the division of multiple sets of data (in this study data are vector's elements) by a common variable in order to negate that variable effect on the data. Therefore, normalization makes the underlying characteristics of the data comparable (Zang et al., 2011). This allows data on different scales to be compared by bringing them to a common scale. In terms of the levels of measurement, these ratios only make sense for ratio measurements (where ratios of measurements are meaningful), not interval measurements (where only distances and not ratios are meaningful). Parametric normalization frequently uses pivotal quantities. Thus, the functions for whose sampling distribution do not depend on the parameters and particularly ancillary statistics; pivotal quantities can be computed from observations, without knowing parameters.

Sorter methodology: The flow chart illustrates that Time Action, Time Stamp and Priority of each job should be multiplied by a specific weight factor (Fig. 1).

- The weigh factor of the Time Stamp is in same manner to the Time Stamp.

- The weigh factor of the time action is changed in an opposite manner to the length of time.

- The amount of weight factor for priority derives from the importance of the six criteria which has been mentioned

For instance, two jobs can be considered. The first one is loading new program on PLC and the second one is summing up two integers with the same sequence but with different time durations received by CCR. The processing of the first job takes one second and the other takes $1 / 1000$ of second. It is not fair that the first job is processed by CCR because of being received just a little bit of time earlier than the second on. This implies that, the other must be delayed 1000 times of its time processing. So, by considering the condition of each job, the multiplied weight factor for that job can be changed. The developed bubble sort is programmed whereby MATLAB Distributed Computing Server (DCS) software, coordinating and executing independent MATLAB $\circledR$ operations simultaneously on a cluster of computers and speeding up execution of huge scheduling problem.

To recapitulate, the total time of process will be declined by applying this sorter. Applying this algorithm, the faults caused by ignoring priority and time action (besides time stamp) in scheduling jobs are eliminated. In turn, this algorithm results in a more reliable system of job allocation. In this regard, a coefficient of $\mathrm{K}(\mathrm{Eq} .3)$ is 
multiplied (*) in total faults of system. The multiplied amount showing the sco;'pe faults (error) is automatically removed from the system. Based on the fact that the amount of $K$ is between 0 and $1(0<\mathrm{K}<1)$, the system faults are reduced. On the other hand, the performance of system obviously increases. This state is proved via Passion distribution in below Eq. 1 and 2:

$f(y)=\frac{\lambda y_{e}^{-\lambda}}{y !}$, fory $=0,1,2$

$\mathrm{F}(\mathrm{Y})=\sum_{\mathrm{i}=0}^{\mathrm{y}} \frac{\lambda^{\mathrm{i}} \mathrm{e}-{ }^{\lambda}}{\mathrm{i} !}$

For example, five jobs arrived in the order described as follows:

Time Stamps: 1, 2, 3, 4, 5

And Time Actions: 2, 4, 1, 5, 3

And Priority: 3, 5, 2, 1, 4

In different circumstances, the weight factors of the mentioned parameters $\left(T_{s}, T_{A}, P_{r}\right)$ are changed in order to obtain the best status. In manufacturing systems, priority plays a more indispensable role. On the other hand, the time action (durations) of jobs are the determined items. Consequently, the weight factors can be defined as below:

$\mathrm{A}=\left(0.3 * \mathrm{~T}_{\mathrm{s}}\right)+\left(0.5 * \mathrm{~T}_{\mathrm{a}}\right)+\left(0.2 * \mathrm{P}_{\mathrm{r}}\right)$

Where:

$\mathrm{A}=$ Value of each job

$\mathrm{T}_{\mathrm{s}}=$ Time stamp

$\mathrm{T}_{\mathrm{a}}=$ Time actions

$\mathrm{P}_{\mathrm{r}}=$ Priority

Eventually, the job queue should be reformed based on the value of each job:

Job1 (1.9), Job2 (3.6), Job3 (1.8), Job4 (3.9), Job5 (3.8)

Job4 (3.9), Job5 (3.8), Job2 (3.6), Job1 (1.9), Job3 (1.8)

Evaluation: To evaluate of the afore-mentioned algorithm, simulation method for numerical test case, mathematical proof are employed. Reforming jobs in new queues eliminates the faults of systems caused by ignoring priority criteria aspects and time action of those jobs.

Simulation: Parallel Computing Toolbox software and MATLAB Distributed Computing Server software enable us to coordinate and execute independent MATLAB ${ }^{\circledR}$ operations simultaneously on a cluster of computers, speeding up execution of large MATLAB jobs.

In some occasions, it is logical not to forward jobs to the machine (even in the case of the non-empty line for that machine) but to wait for the moment that the tense job will be entered the line. Such an approach may be introduced in stochastic scheduling too. A unification of both approaches seems to be more effective than each of them separately (Tsai and Lin, 2003). There is no method for scheduling with considering these three criteria to enhancing the reliability of system. The proposed method for these sensitive industrial systems can provide perfect scope in which the reliability of system will be considerably increased.

In this part, we simulate the algorithm with MATLAB DCS software. So, first we find a resource as a scheduler (Fig. 2 and 3):

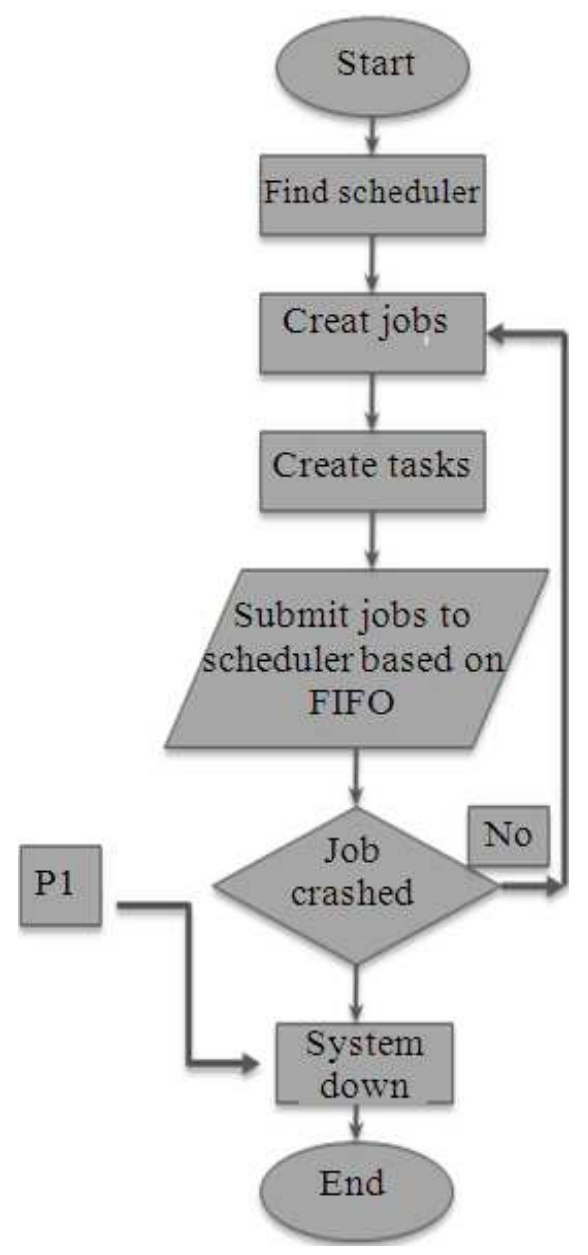

Fig. 2: The flowchart of existing method of scheduling 


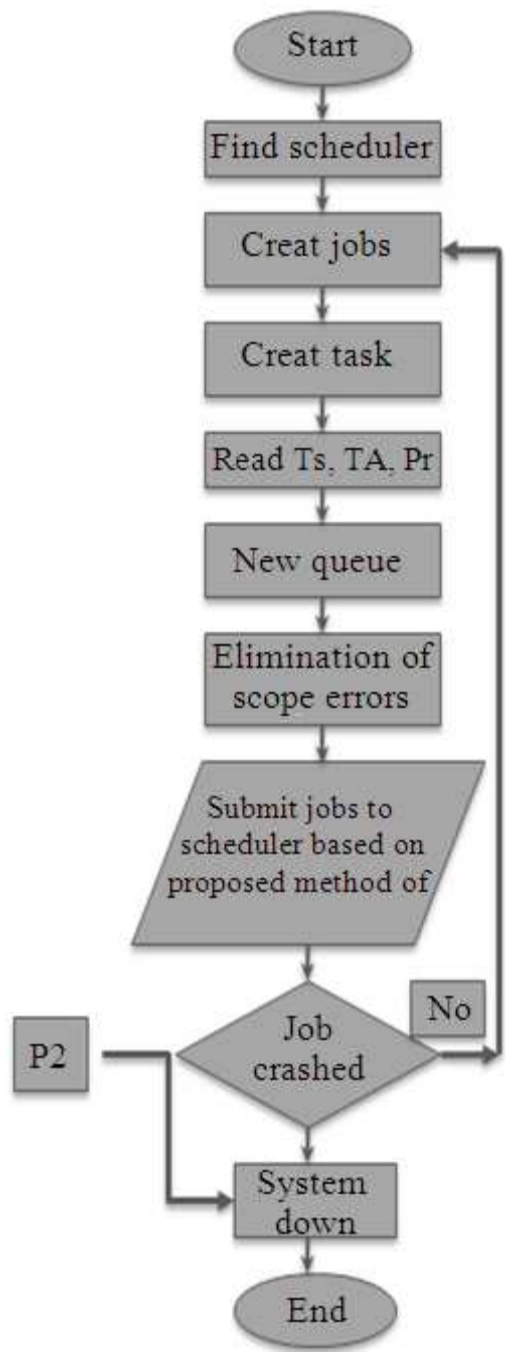

Fig. 3: The flowchart of proposed method of scheduling

sched = find Resource ('scheduler', 'type', 'local') then, we create 3 jobs (job1, job2 and job3):

Job1 = create Job (sched)

Job2 $=$ create Job (sched)

Job3 = create Job (sched)

In third step assigns a task to each of jobs:

Create task (job1, @sum, 1, \{[1 1 1 $]\})$

Create task (job2, @sum, 1, \{[2 2] $\}$ )

Create task (job3, @sum, 1, \{[ [3 3 3$]\}$ )
Submit (job1);

Submit (job2);

Submit (job3);

In the last step we can have the final computing with below instructions:

Wait for state ( $\mathrm{j}$ )

Results $=$ get all output arguments $(\mathrm{j})$

After this simulation, the results can be sorted with applying three important parameters (Time stamp, Time action or time duration and Priority) afterwards new queue will be reformed. By the applying the reformed queue for the system, optimized results will be obtained in result section.

Mathematical proof: To show the reliability enhancement by applying the proposed method of scheduling, in this section a mathematical proof is presented. Based on the simulated algorithm and the following Eq. 3 and 4:

$$
\begin{aligned}
& \mathrm{f}(\mathrm{y})=\frac{\lambda \mathrm{y}_{\mathrm{e}}^{-\lambda}}{\mathrm{y} !}, \text { fory }=0,1,2 \\
& \mathrm{~F}(\mathrm{Y})=\sum_{\mathrm{i}=0}^{\mathrm{y}} \frac{\lambda^{\mathrm{i}} \mathrm{e}-{ }^{\lambda}}{\mathrm{i} !}
\end{aligned}
$$

where, $\lambda$ is the distribution parameter.

Then, cumulative distribution function is:

$$
\mathrm{F}(\mathrm{Y})=\frac{\lambda^{\mathrm{Y}} \mathrm{e}-{ }^{\lambda}}{\mathrm{Y} !}
$$

Where:

$\mathrm{E}_{\text {Scope }}=$ The errors caused by ignoring priority aspects and time action of each job

$\mathrm{E}_{\text {Non-scope }}=$ The errors occurring because of other reasons (these errors are not related to ignoring priority and time action of job) Eq. 5-8:

$$
\begin{aligned}
& \mathrm{E}_{\text {Total }}=\mathrm{E}_{\text {Scope }}+\mathrm{E}_{\text {Non-scope }} \\
& 1: \mathrm{F}\left(\mathrm{E}_{\text {Total }}\right)=\frac{\lambda^{\mathrm{E}}{ }_{\text {Total }} \mathrm{e}-\lambda}{\mathrm{E}_{\text {Total }} !}
\end{aligned}
$$

$$
2: F\left(\mathrm{E}_{\text {scope }}\right)=\frac{\lambda_{\text {Scope }}^{\mathrm{E}} \mathrm{e}-\lambda}{\mathrm{E}_{\text {scope }} !}
$$

scheduler in other word, we submit jobs to the job queue. As we know, here scheduler has a coordinator effect: 
End time Am. J. Applied Sci., 9 (9): 1435-1442, 2012

$$
\mathrm{K}=\frac{\frac{\lambda \mathrm{E}_{\text {scope }} \mathrm{e}^{-\lambda}}{\frac{\mathrm{E}_{\text {scope }} !}{\lambda \mathrm{E}_{\text {Total }} \mathrm{e}^{-\lambda}}}}{\mathrm{E}_{\text {Total }} !}
$$

The $e^{\lambda}$ causes the equation becomes non-linear. In Eq. $6, \mathrm{e}^{\lambda}$ is deleted from the whole fraction. As a result, $\mathrm{k}$ ranges between zero and one.

Probability and reliability relationship can be defined in the formula below Eq. 9:

$\mathrm{R}=1-\mathrm{P}(\mathrm{E})$

Where:

$\mathrm{R}=$ Stands for system reliability

$\mathrm{P}(\mathrm{E})=$ The number of events (errors) in time duration Eq. 10:

$\mathrm{P}(\mathrm{E})=\mathrm{P}\left(\mathrm{E}_{\text {Scope }}\right)+\mathrm{P}\left(\mathrm{E}_{\text {Non-scope }}\right)$

$\mathrm{P}\left(\mathrm{E}_{\text {Scope }}\right)=\mathrm{k} * \mathrm{P}(\mathrm{E})(2)$

where $0<\mathrm{k}<1$.

By combining equations Eq. 7 and 8 mentioned above, $\mathrm{P}(\mathrm{E})$ can be calculated from the formula below Eq. 11 and 12:
$\mathrm{P}(\mathrm{E})=\frac{\mathrm{P}\left(\mathrm{E}_{\text {Non-scope }}\right)}{1=\mathrm{k}}=\frac{1}{1=\mathrm{E}_{\text {Non-scoope }}}$
Or $: \mathrm{k}^{\prime}=\frac{1}{1-\mathrm{k}}$

Then : $\mathrm{P}(\mathrm{E})=\mathrm{K}^{\prime *} \mathrm{E}_{\text {Non-scope }}$

where $\mathrm{k}^{\prime}>1, \mathrm{k}^{\prime}=\mathrm{k}+1$ and $\mathrm{k}$ (or $\mathrm{k}^{\prime}$ ) is systemdependent; it varies from system to another. Since systems are never error-free and encounter errors all the time, $\mathrm{K}$ is always greater than 0 . Based on this justification, the $\mathrm{P}$ ( $\left.\mathrm{E}_{\text {Non-scope }}\right)$ is not equal to 0 . Therefore, the value of $\mathrm{k}$ is always less than 1 .

As mentioned, in the presented algorithm, After performing this simulation for several times and create random function for industrial failures as below it is revealed that applied algorithm result in better performance in terms of reducing the failure of systems. Besides, it is obvious that it will cause increase the reliability of the system via fair judgment and temporary queue. The below Fig. 4 is illustrated that the applied method results in increasing performance.

It is illustrated that the time of crashing server machine based on station and close area. As shown, it is ostensible that efficiency of proposed algorithm is better than previous algorithm. Subsequently, the probability of crashing will be decreased. Thus, our system is reliable and as mentioned, faults occur less than normal status. Also distributed system is more accessible than previous status, so system accessibility increased.

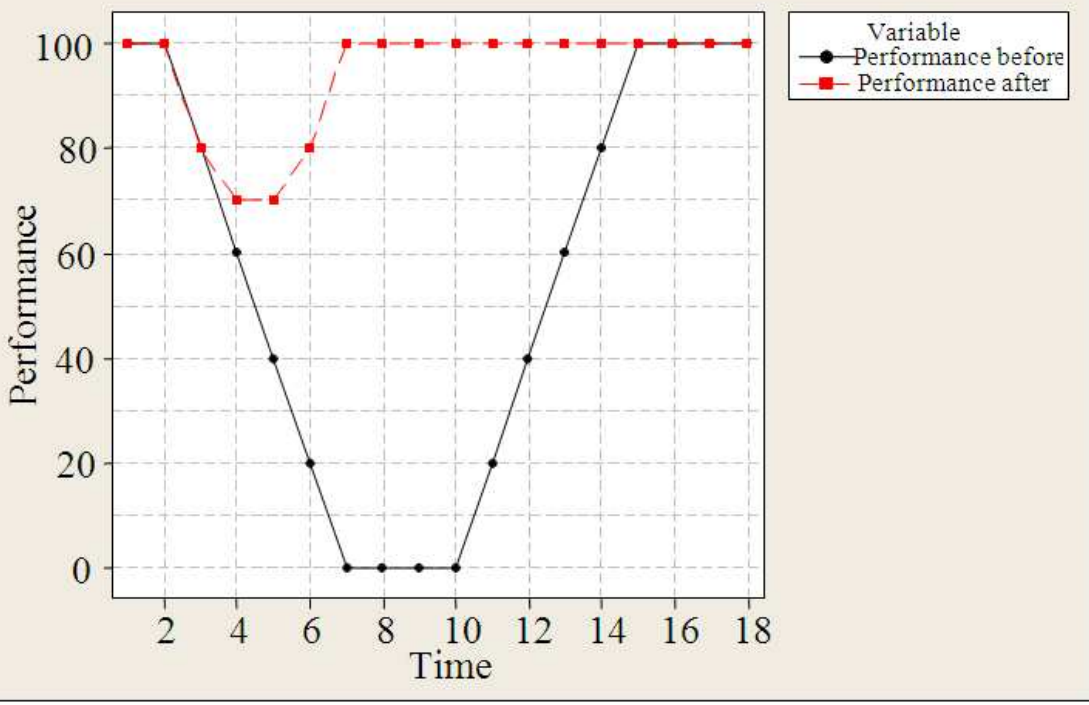

Fig. 4: A comparison between the previous status and the status produced by the proposed algorithm 


\section{CONCLUSION}

To wrap up, based on applying the proposed method of scheduling which have been mentioned. We can come to this conclusion that reliability of systems has been remarkably enhanced. It causes increasing accessibility as well. Besides for future and further study, it is suggested that accomplishing some state-ofthe-art algorithms in order to prevent the starvation of jobs in industrial manufacturing environments.

\section{REFERENCES}

Adamyan, A. and D. He, 2002. Analysis of sequential failures for assessment of reliability and safety of manufacturing systems. Reliab. Eng. Syst. Safety, 76: 227-236. DOI: 10.1016/S0951-8320(02)00013-3

Baker, K.R., 1974. Scheduling a full-time workforce to meet cyclic staffing requirements. Manage. Sci., 20: 1561-1568.

Chen, J.S., 2010. Integration of job scheduling with delivery vehicles routin. Inform. Technol. J., 9: 1202-1206.

Cruz, R.L. and J.T. Tasi, 1996. COD: Alternative architectures for high speed packet switching. IEEE/ACM Trans. Netw., 4: 11-21. DOI: $10.1109 / 90.503758$

Davis, L., 1987. Genetic Algorithms and Simulated Annealing. 1st Edn., Pitman, London, ISBN-10: 0934613443, pp: 216.

Fazlollahtabar, H. and M. Zandieh, 2010. Cost optimization in an AGV-based automated manufacturing system associated with product inspection and machine reliability. Proceedings of the Conference on Engineering Systems Management and Its Applications (ICESMA), Second International, Mar. 30-Apr. 1, IEEE Xplore Press, Sharjah, pp: 1-6.

Holmes, J.W., 1995. A logistical management system using calculated raw-process-time factors for production control. Proceedings of the Conference and Workshop Advanced Semiconductor Manufacturing, Nov. 13-15, IEEE Xplore Press, pp: 127-130. DOI: 10.1109/ASMC.1995.484353

Huafei, Z., Chigan, C.T. and B. Feng, 2006. Turing assessor: A new tool for cyber security quantification. Proceedings of the Conference on Wireless Communications and Networking, Apr. 36, IEEE Xplore Press, Las Vegas, NV, pp: 629633. DOI: $10.1109 /$ WCNC.2006.1683542

Ishikawa, K., 1985. What is Total Quality Control? The Japanese Way. 1st Edn., Prentice-Hall, Englewood Cliffs, N.J., pp: 215.
Kapanoglu, M. and M. Alikalfa, 2011. Learning IFTHEN priority rules for dynamic job shops using genetic algorithms. Robotics Comput. Integr. Manufac., 27: 47-55. DOI: 10.1016/j.rcim.2010.06.001

Krallmann, J., U. Schwiegelshohn and R. Yahyapour, 1999. On the design and evaluation of job scheduling algorithms. Proceedings of the Job Scheduling Strategies for Parallel Processing, (SSPP' 99), ACM Springer-Verlag, London, UK, pp: $17-42$.

Mahdavi, L. and B. Shirazi, 2010. A review of simulation-based intelligent decision support system architecture for the adaptive control of flexible manufacturing systems. J. Art. Intell., 3: 201-219.

Mebarki, N., A. Dussauchoy and H. Pierreval, 1998. On the comparison of solutions in stochastic simulation-optimization problems with several performance measures. Int. Trans. Operat. Res., 5: 137-145. DOI: $\quad 10.1111 /$ j.14753995.1998.tb00109.x

Oguz, C. and M.F. Ercan, 2005. A genetic algorithm for hybrid flow-shop scheduling with multiprocessor tasks. J. Schedul., 8: 323-351. DOI: 10.1007/s10951-005-1640-y

Omar, M.S., 2007. On the solution of the problem of scheduling unrelated parallel machines with machine eligibility restrictions under fuzziness. J. Applied Sci Res., 2: 404-411.

Pierreval, H. and N. Mebaraki, 1997. Dynamic scheduling selection of dispatching rules for manufacturing system. Int. J. Prod. Res., 35: 15751591.

Pinedo, M., 2008. Scheduling: Theory, Algorithms, and Systems. 3rd Edn., Springer, New York, ISBN-10: 0387789340, pp: 671.

Qiao, W. and L. Qiaoyun, 2009. Solving the flexible job shop scheduling problems based on the adaptive genetic algorithm. Proceedings of the International Forum on Computer ScienceTechnology and Applications, Dec. 25-27, IEEE Xplore Press, Chongqing, pp: 97-100. DOI: 10.1109/IFCSTA.2009.30

Rashid, M. and N. Akhtar, 2006. A new multilevel CPU scheduling algorithm. J. Applied Sci., 6: 2036-2039.

Sabin, G., M. Lang and P. Sadayappan, 2007. Moldable parallel job scheduling using job efficiency: An iterative approach. Proceedings of the 12th International Conference on Job Scheduling Strategies for Parallel Processing, (SSPP' 07), Springer-Verlag, Saint-Malo, France, pp: 94-114. 
Samreen, F and M.S.H. Khiyal, 2007. Q-learning scheduler and load balancer for heterogeneous systems. J. Applied Sci., 7: 1504-1510.

Sheyner, O., Haines, J., S. Jha, R. Lippmann and J.M. Wing, 2002. Automated generation and analysis of attack graphs. Proceedings of the IEEE Symposium on Security and Privacy, (SP' 02), IEEE Xplore Press, pp: 273-284. DOI: 10.1109/SECPRI.2002.1004377

Shukla, S.K., Y. Son and M. Tiwari, 2008. Fuzzy-based adaptive sample-sort simulated annealing for resource-constrained project scheduling Int. J. Adv. Manufac. Technol., 36: 982-995. DOI: 10.1007/s00170-006-0907-6

Smith, D.J., 2005. Reliability, Maintainability And Risk: Practical Methods For Engineers. 7th Edn., Butterworth-Heinemann Oxford Boston, ISBN-10: 0750666943, pp: 346.

Taghavifard, M.T., M. Heydar and S.S. Mousavi, 2009. A genetic algorithm for scheduling flexible manufacturing cells. J. Applied Sci., 9: 97-104.

Taiwo, A.S., 2007. Determination of the optimal manpower size using linear programming model. Res. J. Bus. Manage., 1: 30-36.

Tian, J., Cheng, T.C.E., C.T. Ng and J. Yuan, 2009. Online scheduling on unbounded parallel-batch machines to minimize the makespan. Inform. Proc. Lett., 109: 1211-1215. DOI: 10.1016/j.ipl.2009.08.008

Tsai, C.F. and F.C. Lin, 2003. A new hybrid heuristic technique for solving job-shop scheduling problem. Proceedings of the 2nd IEEE International Workshop on Intelligent Data Acquisition and Advanced Computing Systems: Technology and Applications, Sept. 8-10, IEEE Xplore Press, Lviv, pp: 53-58. DOI: 10.1109/IDAACS.2003.1249515
Venkatachalam, S., C. Arumugum, K. Raja and V. Selladurai, 2008. Quality function deployment in agile parallel machine scheduling through neural network technique. Asian J. Sci. Res., 1: 146-152.

Vinod, V. and Sridharan, R., 2008. Development and analysis of fuzzy priority rules for scheduling a dynamic job shop production system. Proceedings of the IEEE International Conference Industrial Engineering and Engineering Management, Dec. 811, IEEE Xplore, Singapore, pp: 1418-1422. DOI: 10.1109/IEEM.2008.4738104

$\mathrm{Wu}$, S. and M.J. Zuo, 2010. Linear and nonlinear preventive maintenance models. IEEE Trans. Reliab., 59: 242-249. DOI: 10.1109/TR.2010.2041972

Zang, R., J. Bai, H. Xu, L. Zhang and J. Yang et al., 2011. Selection of suitable reference genes for realtime quantitative PCR studies in Lanzhou fat-tailed sheep (Ovis aries). Asian J. Sci. Res., 6: 789-804.

Zhang, X., 2010. Job-shop scheduling problems using timed planning. Proceeding of the 4th International Conference on Secure Software Integration and Reliability Improvement Companion (SSIRI-C), Jun. 9-11, IEEE Xplore Press, Singapore, pp: 110117. DOI: 10.1109/SSIRI-C.2010.35 